\title{
O LIVRO DOS QUADRADOS
}

\section{THE BOOK OF SQUARES}

\author{
José dos Santos Guimarães Filho ${ }^{1}$ \\ Universidade Federal do Pará - UFPA \\ João Cláudio Brandemberg ${ }^{2}$ \\ Universidade Federal do Pará - UFPA
}

\begin{abstract}
Resumo
Neste artigo, como uma síntese de nossa pesquisa de dissertação de mestrado, na qual nos dedicamos a estudar a vida do matemático italiano Leonardo Fibonacci (1180 1250) e de uma de suas obras, o Liber Quadratorum (1225). Objetivamos apresentar uma pequena porção da vida desse matemático brilhante do século XIII e de sua obra. Para tanto, iniciamos descrevendo o ambiente em que Leonardo Fibonacci estava inserido, bem como do Liber Quadratorum. Assim, apresentamos o cenário do décimo terceiro século e o seu renascimento comercial, bem como as transformações que este pré-renascimento ocasionou neste período, que influenciaram Leonardo Fibonacci em suas viagens, o levando a se dedicar ao estudo da álgebra geométrica oriental e dos números indo-arábicos, levando a escrever alguns livros, dentre os quis temos o Liber Quadratorum, que teve como motivação um torneio matemático, sendo convidado pelo rei Frederico II, a quem foi dedicado o livro, apresentamos o contexto e as circunstâncias em que foi escrito este livro e ainda neste trabalho os três primeiros problemas contidos no Liber Quadratorum, assim como, suas explicações e desdobramentos, para que o leitor tenha uma dimensão mais adequada do que se trata o livro. É importante evidenciar que nos baseamos principalmente na tradução de L. E. Sigler (1987) e de forma secundária no trabalho de McClenon (1919). Com o exposto nesse trabalho, percebemos que estes problemas ainda são relevantes e possuidores de potenciais para o ensino e para a aprendizagem de matemática, pois, mostram indícios do estudo das ternas pitagóricas de diversas formas.
\end{abstract}

Palavras-chave: Liber Quadratorum; Leonardo Fibonacci; Ternas pitagóricas; Ensino de matemática.

\begin{abstract}
In this paper, as a synthesis of our master's dissertation research, in which we devote ourselves to studying the life of the Italian mathematician Leonardo Fibonacci (1180 1250) and one of his works, the Liber Quadratorum (1225). We aim to present a small portion of the life of this brilliant mathematician of the thirteenth century and his work. To do so, we began by describing the environment in which Leonardo Fibonacci was inserted, as well as the Liber Quadratorum. Thus, we present the scenario of the
\end{abstract}

\footnotetext{
1 js_guima@hotmail.com

2 brand@ufpa.br
} 
thirteenth century and its commercial renaissance, as well as the transformations that this pre-Renaissance brought about in this period, which influenced Leonardo Fibonacci in his travels, leading him to devote himself to the study of oriental geometric algebra and the indo-arabic numerals, going to write some books, among them we wanted the Liber Quadratorum, which was motivated by a mathematical tournament, invited by King Frederick II, to whom the book was dedicated, we presented the context and the circumstances in which it was written this book and still in this work the first three problems contained in the Liber Quadratorum, as well as its explanations and unfoldings, so that the reader has a more adequate dimension of what the book is about. It is important to note that we rely mainly on the translation of L. E. Sigler (1987) and on a secondary form in the work of McClenon (1919). With this work, we realize that these problems are still relevant and have potential for teaching and learning mathematics, as they show evidence of the study of the Pythagorean triples in various ways.

Keywords: Liber Quadratorum; Leonardo Fibonacci; Pythagorean Terns; Mathematics teaching.

\section{Introdução}

Neste artigo trazemos uma síntese de nossa pesquisa de mestrado, em andamento, na qual nos dedicamos a estudar o matemático italiano Leonardo de Pisa ou Pisano mais conhecido por Fibonacci, que neste trabalho optamos por nos referir a ele como Leonardo Fibonacci.

Este matemático elaborou muitas contribuições para as gerações contemporâneas a ele, assim como as posteriores, se valendo de pesquisas e métodos dos estudiosos orientais. No entanto, não apenas se debruçando nessas novas formas de fazer matemática, mas desenvolvendo seus próprios métodos, culminando em obras matemáticas que causam impacto, passando a ser objeto de estudo para muitos pesquisadores.

Percebemos então com uma busca simples, em alguns bancos de dados como o da CAPES (plataforma sucupira), SCIELO (Scientific Electronic Library Online) e ERIC (Education Resources Information Center), que há trabalhos referentes aos livros de Leonardo Fibonacci, principalmente no que diz respeito ao Liber Abaci. Em contrapartida a essa gama de trabalhos referentes ao livro Liber Abaci, não vemos a mesma disposição para o Liber Quadratorum, muito embora tenha sua importância, a exemplo da ótima organização e de uma escrita rica de coleção de teoremas (proposições), a partir de análises envolvendo indeterminados do segundo grau 
(McCLENON, 1919). Dessa forma, entendemos ser válido um trabalho que busque melhor apresentar este livro em língua portuguesa.

Desta forma, direcionamos nossa atenção para este matemático e para uma obra em especifico de sua autoria, o Liber Quadratorum, o qual apresenta proposições ou problemas dignos de serem explorados, haja vista, que Leonardo Fibonacci tem uma maneira peculiar de demonstrar suas ideias.

Iniciamos nossa escrita com uma breve biografia de Leonardo Fibonacci, seguida de três problemas contidos no Liber Quadratorum e suas explicações com notações mais atuais, as quais não eram disponíveis a Leonardo Fibonacci neste momento. Assim, objetivamos nesta comunicação apresentar um pouco desse matemático brilhante do século XIII e de sua interessante obra, o Liber Quadratorum.

\section{Leonardo Fibonacci: contexto histórico e social da vida e obra.}

Elaboramos a escrita desta subseção a partir do uso de um diagrama modelo (figura 1) adequado ao contexto histórico e social do nosso personagem principal e de sua obra. Este diagrama modelo foi elaborado após o estudo preliminar do Liber Quadratorum e dos personagens envolvidos, baseado nas propostas indicado por Chaquiam (2015), publicado no livro História da matemática em sala de aula: propostas para integração dos conteúdos matemáticos e em Mendes e Chaquiam (2016), publicado no livro Histórias nas aulas de matemática: fundamentos e sugestões didáticas para professores. 


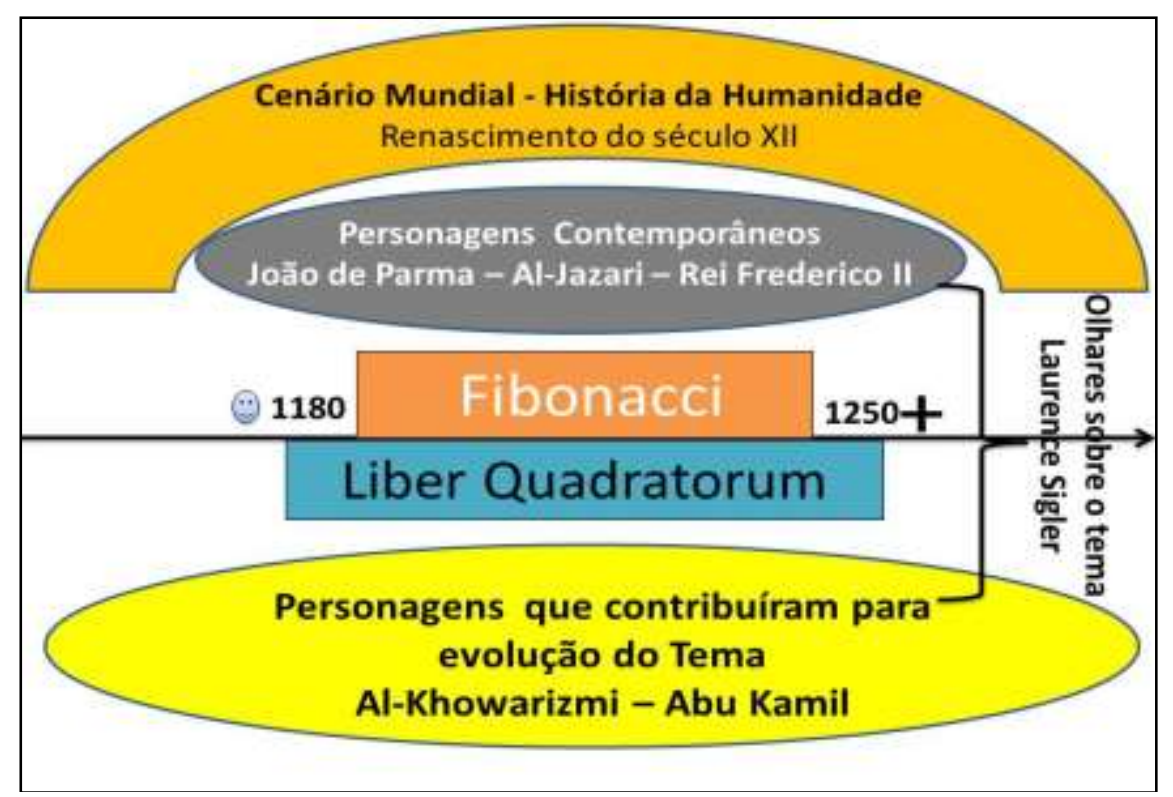

Figura 1 - Diagrama ilustrando o cenário de desenvolvimento do Liber Quadratorum, por Leonardo Fibonacci

Em conformidade como o diagrama proposto iniciamos com o renascimento do décimo segundo século, que advém de um extenso período marcado por instabilidades, quando a Europa passava por transformações e atingia o apogeu do sistema feudal no século XI, na baixa idade média (FRANCO JÚNIOR, 2001). Em meio a essas transformações, de cunho social, político, econômico e cultural, três nos chama a atenção: a transformação na forma de produzir, o crescimento demográfico e o renascimento comercial (SESTITO; OLIVEIRA, 2010).

Nesse contexto de transformações e surgimento das cidades e da burguesia, temos o florescimento do comércio ou a retomada (renascimento) do comércio. Com o aumento da produção agrícola, da produção artesanal urbana e o contato com povos orientais o comércio ganha um expressivo impulso, desenvolvendo rotas locais e internacionais, tanto para o norte quanto para o sul. É nesta ocasião que surgem alguns personagens que influenciaram diretamente ou indiretamente este período do renascimento. Entre estes, um personagem nos chama a atenção, Leonardo Fibonacci que nasceu em 1180 em Pisa na Itália.

Pisa neste momento pré-renascentista, era um centro comercial importante, no qual seu pai Guiglielmo Bonacci, era um secretário da República de Pisa ligado aos negócios mercantis (OLIVEIRA, 2013). Seu pai, um mercador italiano com interesse no norte da África, iniciou os seus negócios com assuntos de contabilidade mercantil, 
despertando também o interesse de Leonardo Fibonacci por matemática, que foi além das aplicações práticas.

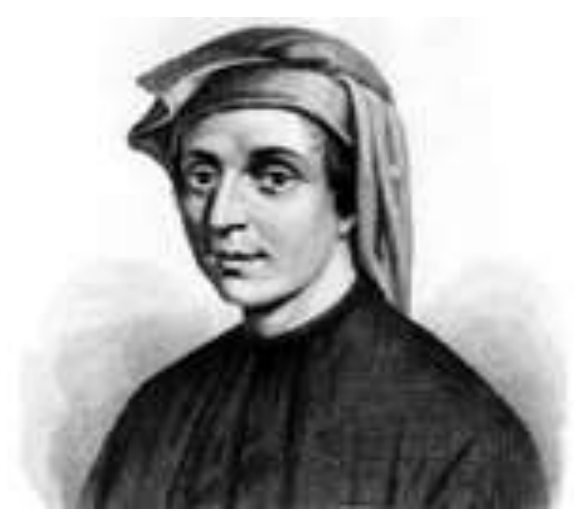

Figura 2 - O jovem matemático Leonardo Fibonacci

Viajando com o seu pai nos últimos anos do décimo segundo século, Leonardo Fibonacci fez uma turnê no Oriente, viu os grandes mercados do Egito e Ásia Menor, foi tão longe como a Síria, e voltou através de Constantinopla. Diferentemente da maioria dos viajantes, Leonardo não se contentou em dar um simples olhar para o estranho e novas paisagens que o rodeava, mas ele estudou cuidadosamente os costumes do povo, e especialmente, procurou instrução no sistema aritmético que estava sendo encontrado de forma tão vantajosa pelos comerciantes orientais (MCCLENON, 1919). Castillo (2007) traz em seu trabalho, que Leonardo Fibonacci nessas viagens estudou sobre a supervisão de um professor árabe (sem citar seu nome). Isso o levou a entrar em contato direto, segundo Eves (2011), com os procedimentos matemáticos orientais e árabes, passando a ser um sério defensor dos números indo-arábicos a partir de conhecêlos nessas viagens.

Leonardo Fibonacci se sente tão motivação a estudar os números indo-arábicos, que seus estudos o levaram a escrever um livro chamado Liber Abaci (o livro do ábaco ou do cálculo) em 1202, logo depois de retornar a sua cidade natal, sendo reeditado em 1228. Essa obra em conformidade com Garbi (2007), torna Leonardo Fibonacci o primeiro cristão a discorrer sobre álgebra na Europa. O livro segundo Boyer (1974), trata de uma maneira mais acentuada de números do que de geometria descrevendo as nove cifras indianas, assim como o símbolo zero. De acordo com Serrão (2014), Leonardo Fibonacci objetivava com este livro propor outro caminho para as realizações de cálculo além do ábaco. 
Posteriormente a essa publicação sua fama se estende pela a Europa e seus estudos contínuos o levaram a criação de seu segundo livro, o qual temos conhecimento. Intitulado de Practica Geometriae (Geometria Prática), que segundo Oliveira (2013) descreve seus conhecimentos de geometria e trigonometria, escrito em 1220. Oliveira (2013) ainda traz em seu trabalho que esta obra é dividida em oito capítulos trazendo uma grande coleção de problemas geométricos de teoremas baseados nos Elementos de Euclides. O livro foi dedicado ao astrônomo Imperial Dominicus Hispanus, o qual lhe apresentou ao Imperador Frederico II.

Sua terceira publicação foi escrita no ano de 1225, um tratado intitulado Flos (Flor) em que há problemas indeterminados que lembram Diofante e problemas determinados que lembram Euclides, os árabes e os chineses (BOYER, 1974). Nesse período é chamada a atenção do rei Frederico II pela repercussão causada pelas obras e conhecimento de Leonardo Fibonacci, enviando-lhe um convite para participar de um torneio matemático, o qual resulta em sua quarta obra o Liber Quadratorum publicado no mesmo ano do livro Flos.

\section{Apresentação do Liber Quadratorum}

O Liber Quadratorum, que segundo Oliveira (2013) significa "livro dos quadrados", carrega em seu conteúdo a teoria dos números que dentre outros, examina o método para encontrar as ternas pitagóricas e Serrão (2014), cita que é o livro mais extenso escrito por Leonardo Fibonacci. Faz-se necessário saber, que esta obra advém de um torneio matemático que Leonardo Fibonacci participou a convite do rei Frederico II.

Nesse torneio foram propostos três problemas dentre os quais o mais famoso é o primeiro, onde Leonardo Fibonacci é desafiado a encontrar um número quadrado que quando somado ou subtraído cinco continua sendo um quadrado de um número racional, (CASTILLO, 2007). Em acordo com Boyer (1974), tanto a resposta (41/12) quanto a solução são encontrados no Liber Quadratorum na proposição dezessete. Os trabalhos de Leonardo Fibonacci, segundo Oliveira (2013), trazem uma forte influência dos matemáticos al-Khwârizmî (708 - 850) e Abû Kâmil (850 - ???) entre outros mestres árabes. Para dar uma ideia do conteúdo deste trabalho notável, apresentaremos algumas proposições que este livro contém, na mesma ordem encontrada no original. 
Leonardo Fibonacci inicia seu livro com uma pequena introdução expondo um de seus pensamentos a respeito do crescimento dos números impares com os números quadrados, que passa a ser a ideia central o que desencadeia as proposições ou problemas contidos no livro,

\begin{abstract}
Eu pensei sobre a origem de todos os números quadrados e descobri que eles surgiram da sequência crescente de números impares; para a unidade é um quadrado, e disso é feito o primeiro quadrado, chamado 1; para essa unidade é adicionado 3, formando o segundo quadrado chamado 4, com raiz 2; se para a soma é adicionado o terceiro número ímpar, chamado 5 , o terceiro quadrado é criado, chamado 9 , com raiz 3 ; e portanto somas de números ímpares consecutivos e a sequência de quadrados sempre crescem juntas em ordem (FIBONACCI, 1225, apud, SIGLER, 1987, p. 4. Tradução nossa).
\end{abstract}

Posteriormente a essa introdução, o autor parte para a apresentação e demonstração de vinte e quatro proposições que compõe o Liber Quadratorum, dos quais traremos apenas três. É interessante dizer que Leonardo Fibonacci para as suas demonstrações entende números como seguimentos de retas e que apresentaremos estas demonstrações com uma notação atual, a qual Leonardo Fibonacci não dispunha no momento.

Proposição 1: Encontrar dois números quadrados cuja soma seja um número quadrado.

Leonardo Fibonacci comenta: Para encontrar dois números quadrados cuja a soma seja um número quadrado, eu devo pegar qualquer quadrado ímpar e eu devo têlo como um dos dois quadrados mencionados; o outro eu encontro em uma soma de todos os números ímpares da unidade até o quadrado ímpar tomado.

Em notação moderna teríamos: Admitindo $2 n+1=x^{2}$, temos que, $1+3+$ $\cdots+(2 n-1)+x^{2}=n^{2}+(2 n+1)=(n+1)^{2}$.

Proposição 2: Qualquer número quadrado excede o quadrado imediatamente anterior pela soma das raízes.

Leonardo Fibonacci comenta: Eu encontrei que qualquer quadrado excede o quadrado imediatamente anterior pela soma das raízes desses quadrados. Por exemplo, 121, do qual a raiz é 11, excede 100, do qual a raiz é 10, pela soma de 10 e 11, nomeados pela soma das próprias raízes. É por isso que um quadrado excede o segundo antes dele pela quantidade que é quatro vezes a raiz do quadrado que está 
entre eles, como 121, que excede 81 por quatro vezes 10; e portanto pode ser encontrado diferenças entre os quadrados pelas distâncias entre as próprias raízes.

Leonardo quis mostrar que, se tomamos $121=11^{2}$ e $100=10^{2}$, teremos que $121=100+11+10$, ou seja, de 100 até 121 temos o intervalo de $21=11+10$, que são a soma das raízes quadradas de 100 e de 121. Leonardo Fibonacci apresenta uma explicação geométrica para esta proposição.

Exemplo geométrico: se tomamos um segmento de reta $\overline{a g}$ com mais dois pontos que são $b$ e $d$ contidos neste seguimento, temos que:

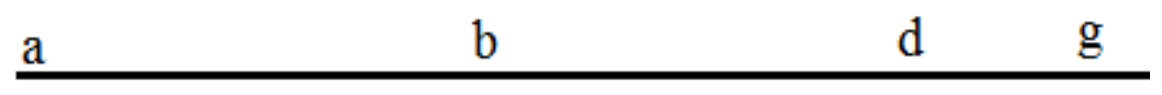

\section{Com isso temos:}

$\checkmark \overline{a b}$ e $\overline{b g}$ são consecutivos;

$\checkmark \overline{b g}-\overline{d g}=\overline{b d}$;

$\checkmark \overline{b d}=\overline{a b}$;

$\checkmark \overline{d g}=1$.

Com estas questões preliminares, Leonardo Fibonacci apresenta a seguinte relação: $\overline{b g}^{2}=\overline{b d} \times \overline{b d}+\overline{d g} \times \overline{d g}+2 \overline{b d} \times \overline{d g}$, onde $\overline{d g} \times \overline{d g}+2 \overline{b d} \times \overline{d g}$ compõe a diferença de um quadrado para outro. Se admitirmos $\overline{a b}=7, \overline{b g}=8$ e $\overline{d g}=1$, temos que,

$$
\begin{gathered}
\overline{b g}^{2}=\overline{b d} \times \overline{b d}+\overline{d g} \times \overline{d g}+2 \overline{b d} \times \overline{d g} \\
8^{2}=7 \times 7+1 \times 1+2 \times 7 \times 1 \\
8^{2}=49+15=64
\end{gathered}
$$

Percebemos que $15=7+8$, no qual a soma das raízes é a diferença entre os quadrados como queríamos mostrar. Vale ressaltar que esta proposição contem dois desdobramentos.

$1^{\circ}$ Desdobramento: se escolhemos o maior quadrado de três números quadrados consecutivos, como $9^{2}, 10^{2}$ e $11^{2}$, teremos que, o maior quadrado será o menor mais o quádruplo da raiz do quadrado médio, assim, temos $11^{2}=9^{2}+(4 \times 10)$.

$2^{\circ}$ Desdobramento: se admitimos um número quadrado impar como o $9 \mathrm{e}$ multiplicarmos por quatro, temos 36 , e tomando o quadrado do antecessor de 9 , temos $8^{2}=64$ e agora somando o quadruplo do quadrado impar escolhido mais o seu antecessor encontramos o quadrado do seu sucessor, logo, $36+64=100=10^{2}$. 
Proposição 3: Existe outra forma de encontrar dois quadrados que formem um número quadrado com sua soma.

Leonardo Fibonacci comenta: Há de fato outra forma de encontrar dois quadrados que formem um número quadrado com sua soma, e isso se encontra no livro $X$ de Euclides. Juntando dois números quadrados, ambos pares ou ambos ímpares, $\overline{a b} e$ $\overline{b g}$; então a soma $\overline{a g}$ será par. Deixe $\overline{a b}$ ser maior que $\overline{b g}$, e $\overline{a g}$ é dividido em duas partes iguais por d. O número $\overline{a d}$ é então um número inteiro por ser metade do número $\overline{a g}$. E se subtrai $\overline{a d}$ do número $\overline{a b}$; restará o número inteiro $\overline{d b}$.

E pelo número $\overline{a g}$ ser dividido em duas partes iguais por $d$, e em partes diferentes por $b$, o produto de $\overline{a b}$ e $\overline{b g}$, mais o quadrado do número $\overline{d b}$, será igual ao quadrado do número $\overline{d g}$; mas aquele que é feito de $\overline{a b}$ vezes $\overline{b g}$ é um quadrado, como $\overline{a b}$ e $\overline{b g}$ são quadrados; aquele que é feito pelo número $\overline{d b}$ vezes $\overline{d b}$ é um quadrado, $e$ portanto são encontrados dois quadrados com a soma de um número quadrado, nomeado o número $\overline{d g}$. Isso que tinha de ser feito.

Exemplificando temos:

$$
\overline{a b} \times \overline{b g}+\overline{d b}^{2}=\overline{d g}^{2}
$$

Para a nossa pesquisa, utilizamos como texto base para as proposições o livro The Book of Squares, que tem como autor L. E. Sigler (1987), pois julgamos ser mais completo, organizando as proposições na mesma ordem de Leonardo Fibonacci. Utilizamos de forma secundária para as proposições e para a biografia de Leonardo Fibonacci os seguintes autores: McClenon (1919) e Castillo (2007).

\section{Considerações finais}

Percebemos com exposto, que estes problemas são ricos, possuidores de potenciais para o ensino e para a aprendizagem, pois mostram alguns indícios como a exploração das ternas pitagóricas de diversas formas. Possibilitando propostas para o currículo de matemática. Assim, entendemos que a exploração criativa dos textos matemáticos históricos podem fazer contribuições para o encaminhamento conceitual e didático de noções das componentes curriculares (LOPES, 2017). Logo examinar situações específicas com um aprofundamento consistente, como no Liber Quadratorum, pode viabilizar respostas mais satisfatórias para o ensino. 
Nesse sentido, nos propomos a explorar as demais proposições contidas neste livro tão valioso para o século XIII e suas possíveis potencialidades, dando continuidade em nossas pesquisas.

\section{Referências}

BOYER, C. B. História da Matemática. Tradução: Elza F. Gomide. São Paulo, SP: Edgard Blücher, 1974.

CASTILLO, R. M. Fibonacci: El Primer Matemático Medieval. 2ª ed. Coleção - La matemática em sus personajes. Espaha: Nivola, 2007.

CHAQUIAM, M. História da Matemática em Sala de Aula: Proposta para Integração aos Conteúdos Matemáticos. SBHM - 2015.

EVES, H. Introdução à História da Matemática. $5^{a}$ ed. - Tradução Hygino H. Domingues. Campinas, SP: Editora da Unicamp, 2011.

FRANCO JÚNIOR, H. A Idade Média: nascimento do ocidente. $2^{\mathrm{a}}$ ed. rev. e ampl. São Paulo, SP: Brasiliense, 2001.

GARBI, G. G. O Romance das Equações Algébricas. 2a ed. São Paulo, SP: Ed. Livraria da Física, 2007.

LOPES, G. L. O. Criatividade Matemática de John Wallis na obra Arithmetica Infinitorum: Contribuições para o ensino de cálculo diferencial e integral na licenciatura matemática. (Tese de Doutorado). UFRN, 2017

MCCLENON, R. B. Leonardo of Pisa his Liber Quadratorum. The American Mathematical Monthly, Vol. 26, No. 1. pp. 1-8. Jan., 1919.

MENDES, I. A.; CHAQUIAM, M. História nas aulas de Matemática: fundamentos e sugestões didáticas para professores. Belém: SBHMat, 2016.

OLIVEIRA, J. J. Sequências de Fibonacci: possibilidades de aplicações no ensino básico. UFBA. Salvador, BA, 2013.

SERRÃ̃, M. M. Problemas Matemáticos da Antiguidade como Estratégia para o Ensino de Matemática na Educação Básica. (dissertação de mestrado). Belém, PA: 2014.

SESTITO, E. A. B.; OLIVEIRA, T. As Transformações do Pensamento na Baixa Idade Média e as Mudanças na Arte. Londrina, 2010.

SIGLER, L. E. The Book of Squares. An annotated translation into modern English. Academic Press:USA, 1987. 